\title{
Dispersión de las Partículas de Emisiones Industriales en el Problema de Optimización Semi-infinita del Área Contaminada
}

\author{
Alina Fedossova ${ }^{1}$, Valeriy V. Fedosov ${ }^{2}$ y Rodrigo A. Britto ${ }^{3}$ \\ (1) Facultad de Ciencias, Universidad Nacional de Colombia, Departamento Matemáticas, Calle 44 No. $45-$ \\ 67 Bogotá - Colombia (afedosova@unal.edu.co) \\ (2) Investigador independiente, Michurinskiy pr., 25-4-242, Moscú - Rusia (vlr.fdsv@gmail.com) \\ (3) Facultad de Administración, Universidad de los Andes, Calle 21 No. 1-20, Bogotá - Colombia \\ (ro-britt@uniandes.edu.co).
}

Recibido Abr. 26, 2018; Aceptado Jun. 28, 2018; Versión final Ago. 8, 2018, Publicado Feb. 2019

\begin{abstract}
Resumen
Este trabajo se propone minimizar los costos de conflicto entre las normas que regulan la calidad del aire y la actividad industrial, que puede ser modelado como un problema de optimización semi-infinita. Para resolver este problema se utilizó el método estocástico de aproximaciones externas en MATLAB, junto a un parámetro de composición y dispersión de partículas, reagrupando el área de distribución de contaminación inicial en subáreas reducidas. El conjunto de fuentes de contaminación se reemplazó por fuentes virtuales para resolver convenientemente el problema original. Los experimentos numéricos permitieron construir mapas de contaminación precisos según la composición no uniforme de las partículas. Así, se encontraron buenas soluciones acorde a las normas ambientales en cada punto de las diferentes zonas.
\end{abstract}

\section{Dispersion of Particles of Industrial Emissions in the Problem of Semi-infinite Optimization of Contaminated Area}

\begin{abstract}
This objective of the work described in this paper is to minimize conflict costs between air pollution regulations and industrial activity which can be modeled as a semi-infinite optimization problem. The problem was solved by using the stochastic method of outer approximations in MATLAB and by introducing particle composition and dispersion as a key parameter, the initial pollution distribution area was regrouped into smaller subareas. Then, the set of pollution sources was replaced with a set of virtual sources, which allows solving the problem in a more appropriate manner by preserving the initial coordinates of the pollution sources. The numerical experiments allowed to build precise pollution maps according to the dispersion of polluting particles. This method provided good solutions that satisfy the safety regulation in each point of different zones.
\end{abstract}

Keywords: air pollution; semi-infinite programming; particulate matter; outer approximation; stochastic algorithms 


\section{INTRODUCCIÓN}

La polución del aire ha ganado gran protagonismo debido a los fuertes efectos negativos sociales y económicos que conlleva, no solo por presentar un riesgo para la sostenibilidad del planeta, sino, por influir en factores de salubridad y productividad. La polución en el aire causa alrededor del $6 \%$ de la mortalidad total anual en los Estados Unidos (Künzli et al., 2000). De forma similar, la presencia de partículas contaminantes en aire incide negativamente en la productividad. Por ejemplo, la investigación de Graff Zivin y Neidell (2011) encontró que altos niveles de concentración de contaminantes de ozono en el aire afectan negativamente la capacidad productiva agrícola en el Valle de California y una reducción de 10 ppb significaría un incremento en la productividad del 5\%, es decir, alrededor de 700 millones de USD ahorrados por las empresas en gastos de mano de obra. De ahí, que en las agendas de políticas públicas se tengan en cuenta los problemas de ecología industrial. El conflicto entre el exceso de emisiones contaminantes causadas por la producción industrial y las responsabilidades de ecología ambiental se ha abordado mediante el diseño de nuevos métodos y forma de reducción de conflictos, un ejemplo son los esfuerzos del gobierno chino por reducir las párticulas sólidas PM 2.5 en un $20.5 \%$. Sin embargo, el panorama de la contaminación ambiental no da signos de mejoría significativa. Solo en el 2015, entre 3.7 y 4.2 millones de personas murieron a causa de la baja calidad del aire. Las estimaciones indican que la polución ambiental será la principal fuente de mortalidad infantil para el 2050 (Duque, 2016).

Si bien en América Latina los programas de reducción de contaminación aún no están completamente desarrollados, han adquirido especial relevancia los estudios relacionados con la polución en el medio ambiente. En ciudades como Ciudad de México, Santiago de Chile, Sao Paulo y Río de Janeiro, los niveles de contaminación para $\mathrm{PM} 10$ y $\mathrm{O}_{3}$ están por encima de los límites permitidos. Sin embargo, la mortalidad asociada a la polución del aire es relativamente baja (Gouveia et al., 2018). Aunque hay otros lugares, por ejemplo, Riohacha, La Guajira, Colombia donde la concentración de PM10 y PST no violan los estándares locales ni los estadounidenses (NAAQS), pero sí se encuentran elementos contaminantes en partículas inhalables (Rojano, Arregoces y Restrepo, 2014). Dentro del control de emisiones contaminantes es importante considerar tanto el nivel máximo permitido de la concentración de la polución como la probabilidad de que este máximo no sea alcanzado ni excedido (Fedosov y Fedossova, 2018; Kohn, 1972). Kohn (1972), basado en un estudio de una sola variable estocástica (velocidad promedio del aire), determinó que el costo de controlar la polución se eleva a medida que la probabilidad de alcanzar los niveles máximos permitidos también incrementa. Por lo general, se consideran las emisiones de tipo definido (gas, polvo, goteo) y su composición (que se mide por parámetros cuantitativos como peso o volumen). Para el manejo del área de contaminación se utilizan los valores de emisiones máximas permitidas (EMP), los cuales se miden con los indicadores brutos (t /año) (Normas Gost, 2015).

Un estudio más profundo de los conflictos descritos usa algoritmos de optimización de contaminación en los modelos de distribución de esta. Por ejemplo, Vaz y Ferreira (2009); y Fedosov y Fedossova (2014) utilizaron modelos de programación semi-infinita de distribución asociada con las coordenadas del área. Desde esta perspectiva, los algoritmos estocásticos de aproximaciones externas proporcionan una búsqueda rápida y efectiva de la infracción de las normas de contaminación y permiten llegar a las soluciones conformes con las regulaciones correspondientes en cada punto del área de control (Fedosov, Fedossova y Buitrago, 2018; Volkov y Zavriev, 1997). Este tipo de algoritmos también funciona de forma adecuada para el caso de funciones de varios extremos. Por ejemplo, Gómez, Fedossova y Britto (2017) utilizan un modelo de minimización de costos de polución en una región tridimensional, basado en programación semi-infinita y usando el método de aproximaciones externas. Este modelo permite localizar fuentes específicas de polución en un área determinada, así como estimar los efectos de estas fuentes sobre el medioambiente.

La construcción de modelos de dispersión de la contaminación de partículas es un tema complejo, y se encuentra en la intersección de varias disciplinas, además normalmente contiene una simplificación, permitida por el investigador. Bajo la influencia aerodinámica y atmosférica, entre otras, las zonas de dispersión de la contaminación son particularmente sensibles al tamaño de las partículas. Las corrientes industriales estacionarias de polvo de fuentes contienen hasta un $40 \%$ de fracciones de fase sólida con dimensiones menores a 2.5 micras (PM 2.5), lo que prácticamente corresponde al límite superior del rango nano (Zaitseva et al., 2013). La contabilidad de la dispersión de los flujos de polvo es necesaria para estimaciones más precisas de las zonas de influencia de esas fuentes y de exposición de la población a ellas (Zaitseva et al., 2013). La dependencia de la distancia de dispersión de la contaminación es inversa al tamaño de las partículas. La dispersión fija de partículas en forma escalar es muy condicional y en realidad no existe, por lo tanto, se aceptan mediciones de intervalos de la composición de partículas. En la práctica, la dispersión se caracteriza por los diagramas de los rangos de tamaño de partícula, y en teoría, por funciones continuas. 
Este trabajo soluciona el problema de minimización de los costos de conflicto entre las normas ecológicas y las industrias y constituye un aporte a la literatura en este campo porque por primera vez se incluye un parámetro que tiene en cuenta la composición de partículas contaminantes y su dispersión. De esta forma la optimización propuesta también controla la propagación de los contaminantes, lo que permite tener soluciones de mayor calidad, colaborando así con los objetivos mundiales de reducción de la polución. Los experimentos numéricos muestran la dependencia de los mapas de contaminación óptimos de la composición de dispersión de las emisiones y la posibilidad de usarla como parámetro de control. La programación, los cálculos y los gráficos se realizaron en MATLAB.

\section{DEFINICIÓN DEL PROBLEMA Y ALGORITMO}

Los contaminantes son partículas de diferentes tamaños, por ejemplo, se pueden medir en micras $\mu$ m, y se caracterizan por tener una composición dispersa en forma de diagramas. Si, por ejemplo, hay diferentes partículas con dimensiones en el rango de $40 \pm 250 \mu \mathrm{m}$, dicho rango se puede distribuir en varios intervalos (por ejemplo, 9). Si se considera una muestra de $100 \mathrm{~g}$, entonces, es posible determinar la masa de las partículas en cada uno de los 9 intervalos. Así, se obtiene un diagrama de la distribución de partículas por masa en porcentajes. Considerando el 100\% como 1, el diagrama estará en fracciones (por ejemplo, la fracción de partículas en el intervalo 1 - 0.09, en el intervalo 2 - 0.15, en el intervalo 3 - 0.22, etc., hasta el último noveno intervalo). Por lo tanto, se consideran las diferentes distribuciones de partículas por fracciones de peso, que pueden unirse a diferentes tamaños de partículas. De esta forma, no es necesario dar tamaños de partículas específicos. Además, los rangos específicos pueden ser arbitrarios. Es importante considerar la teoría de la discusión y no solamente el caso particular de contaminación de una fábrica específica.

En este estudio, se consideran 3 distribuciones características de partículas (Figura 1) con predominio: partículas más grandes - 1 , partículas de tamaño mediano - 2 y partículas pequeñas - 3 . Estas distribuciones se encuentran en la figura 1 en 9 intervalos con una suma igual a 1. Más adelante, en cálculos, 9 intervalos fueron reemplazados por 3 por simplicidad.

La distribución de partículas, según el tamaño, puede ser decreciente, creciente o extrema, pero no siempre es lineal. Si se conoce la composición dispersa de las partículas, es fácil determinar las fracciones de masa de los intervalos de dispersión contabilizados. En el caso de las funciones, los intervalos de dispersión se determinan usando la integración. En la Figura 1 se ven posibles distribuciones de la composición dispersa de partículas de emisión. Las sumas de fracciones (por peso o volumen) para 9 estimaciones son iguales a 1. Las distribuciones muestran el predominio de partículas grandes, medianas o pequeñas en las emisiones.

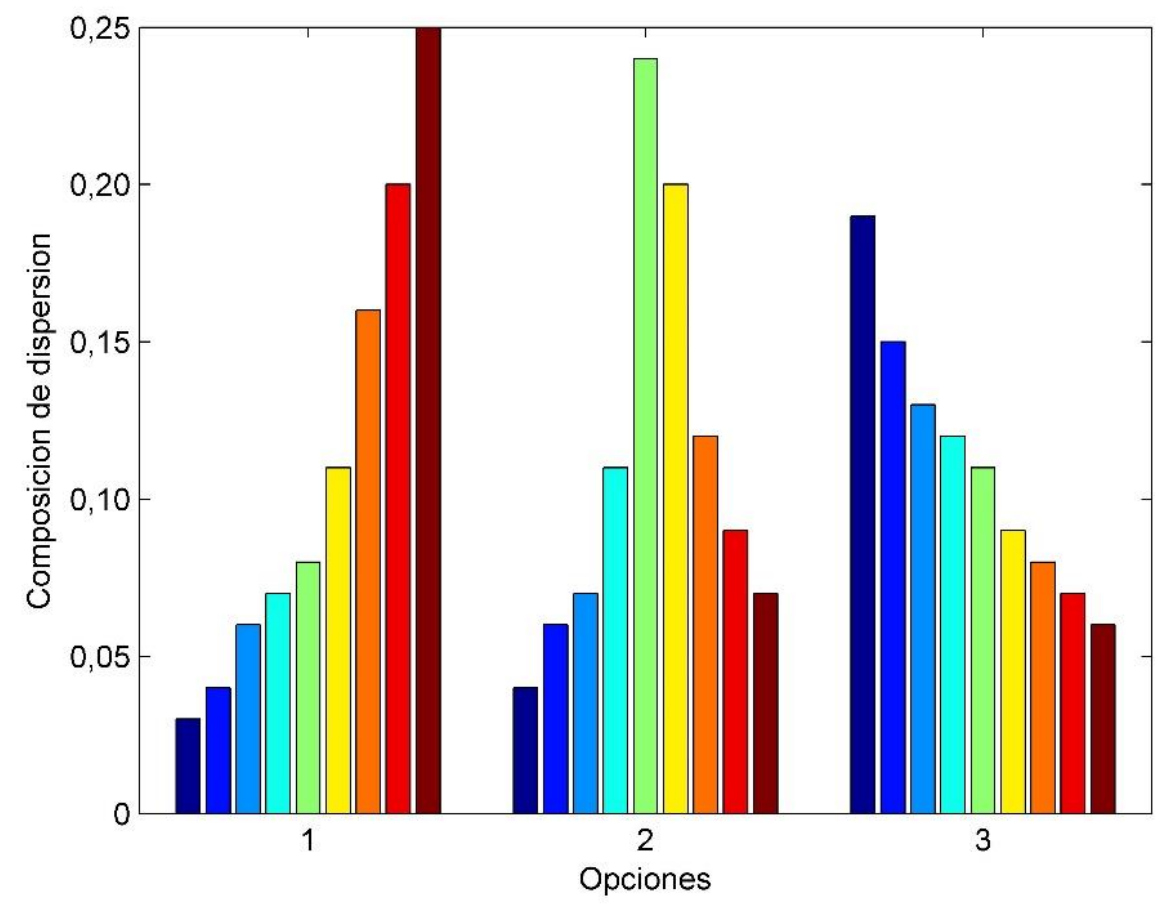

Fig. 1: Composición de dispersión de las partículas en las opciones 1-3.

El dominio bidimensional (territorio) de tamaño $d$ se divide en $\Omega$ zonas. Los límites de las zonas pueden tener configuraciones regulares o curvilíneas. En las zonas se aplican diferentes estándares de contaminación 
Norm. En el área se encuentran $N$ fuentes puntuales de emisiones con coordenadas $\mathrm{tN}$. Las emisiones corresponden solo a un tipo de polución y una variante de la composición dispersa.

Las emisiones de impurezas fueron descritas utilizando las funciones parabólicas pues estas son compactas, todos sus parámetros corresponden a la naturaleza de la dispersión de partículas por una fuente puntual y, además, permiten introducir complicaciones. A un punto $s$ con las coordenadas $[X, Y]$ de cada fuente, viene la contaminación $\mathrm{h}_{s, j}$ dada por la ecuación 1 .

$\mathrm{h}_{s, j}=-a \mathrm{R}_{s, j}^{2}+H_{j}$

Donde: $j$ - es el número de fuente en el conjunto, $H$ - es el vector de potencias de fuentes, $a$-el coeficiente de parábola, $\mathrm{R}$ - es matriz de distancias de fuentes de puntos de área de acuerdo con la ecuación 2.

$\mathrm{R}=\sqrt{\left(\mathrm{tN}_{1, j}-X\right)^{2}+\left(\mathrm{tN}_{2, j}-Y\right)^{2}}$

Donde: $\mathrm{t} \mathrm{N}$ - es la matriz de coordenadas de las fuentes. La fuente de emisión $j$ se considera activa en el punto $s$, si para todo el radio de emisiones para la fuente $\mathrm{R}_{j}^{\max }$ se cumple la desigualdad indicada en la ecuación 3.

$$
\mathrm{R}_{s, j}<\mathrm{R}_{j}^{\max }=\sqrt{\mathrm{H}_{j} / a}
$$

La función de la contaminación general de la región es la imposición de funciones de dispersiones locales de $\mathrm{N}$ fuentes. En el caso de que las fuentes de emisión estén muy separadas, la función general será multiextremal. Se asumió que se conoce la distribución de partículas de contaminación según su tamaño. Se asignaron los diferentes rangos $U$ de la dispersión y se determinó la fracción (en masa) de las partículas $\mu_{u}$ en los rangos contables, los cuales pueden no ser los mismos para la dispersión en ancho. En el caso de una función de dispersión parabólica, el volumen o la masa de la emisión de contaminación por una fuente corresponde al volumen de un paraboloide definido en la ecuación 4.

$V_{j}=\frac{\pi H_{j}^{2}}{2 a}$

Donde: $\mathrm{V}_{\mathrm{j}}$ es el volumen de dispersión de contaminación por fuente $j$.

La introducción en el modelo $U$ rangos de dispersión de partículas se basa en el reemplazo de $N$ fuentes de emisión por $N U$ fuentes virtuales. La suma de los volúmenes de paraboloides virtuales de la extensión $w_{j, u}$ es equivalente al volumen del paraboloide de la fuente sin tener en cuenta la dispersión de las partículas de acuerdo con la ecuación 5.

$V_{j}=\sum_{u=1}^{U} w_{j, u}, \quad y \quad w_{j, u}=\mu_{u} V_{j}$,

Donde: $\mu_{u}$ son partes ponderadas de partículas en los rangos de dispersión contabilizados. Cada una de las fuentes virtuales $N U$ está asociada con uno de los rangos contados $U$ como se indica en la ecuación 6 .

$$
\mu_{u} V_{j}=\frac{\pi H 1_{j, u}^{2}}{2 A_{u}}
$$

Donde: $H 1$ son potencias de las fuentes virtuales $U N$ y $A$ son los coeficientes de la parábola de dispersión de partículas en los intervalos ajustados. Si $\mu$ y $A$ están dados, entonces desde (4) y (5) potencias de los paraboloides virtuales $H 1$ son los que se indican en la ecuación 7.

$$
\mathrm{H} 1_{\mathrm{j}, \mathrm{u}}=\mathrm{H}_{\mathrm{j}} \sqrt{\mu_{\mathrm{u}} \frac{\mathrm{A}_{\mathrm{u}}}{\mathrm{a}}}
$$

En los experimentos numéricos la matriz $H 1[N, U]$ se reemplaza por el vector $H 2[N U]$, y el vector $A[U]$ por el vector $A[N U]$, que significa el cambio de $j=[1, N]$ a $j=[1, N U]$. Se considera brevemente el problema de optimización semi-infinita de emisiones de contaminación, considerando las normas permitidas de polución 
(Vaz, 2009; Normas GOST, 2014). Para ello en la ecuación 8 se presenta un problema clásico de programación matemática.

$$
f_{x} \rightarrow \min _{x \in X^{0}} C_{x}, g_{x, y} \leq 0, \forall_{y} \in Y^{0},
$$

Dónde: $f(x)$ y $g(x, y)$ son funciones continuamente diferenciables sobre compactos convexos $X^{0}, X^{0} \times Y^{0}$ y el conjunto $Y^{0}$ plantea el sistema finito de restricciones. Si $Y^{0}$ define coordenadas espaciales y dado un conjunto infinito de puntos en el área, se llega a tener el conjunto infinito de restricciones y se define el problema de optimización semi-infinita (SIP). En el problema (8) el vector $x$ define los porcentajes de reducción de contaminación por fuentes.

\section{El Método Estocástico de Aproximaciones Externas}

Para resolver este tipo de problemas (SIP) lo importante es reemplazar el conjunto infinito de restricciones por uno finito. Uno de los métodos avanzados y recientes para solucionar este tipo de problemas es el método estocástico de aproximaciones externas (Volkov y Zavriev, 1997), construido a partir del mecanismo de las funciones y conjuntos cuasi-óptimos y del esquema de la búsqueda estocástica activa de las restricciones críticas. El método está bien estudiado en la parte de su convergencia y aplicabilidad, por ejemplo, para el problema famoso de aproximación de Chebyshev. Su idea principal es llevar el problema original SIP a una serie de problemas aproximadas finitos de optimización buscando cada vez las restricciones relevantes críticas y a la vez controlando el crecimiento de su cantidad. La cantidad de restricciones críticas en los problemas de ecología industrial suele ser finita y se determina con el número de máximos locales de la función general de exceso de emisiones de fuentes sobre las normas del área. Para ampliación de los detalles de algoritmo consultar Volkov y Zavriev, 1997; Gómez, Fedossova y Britto , 2017. La sustitución del problema SIP por una secuencia de problemas finitos lleva a una matriz compacta de programación no lineal (a la cual no llegan todas las restricciones sino solamente las críticas) donde se observa el incumplimiento

En general las etapas involucradas en el algoritmo estocástico de aproximaciones externas generalmente primero utilizan un procedimiento estocástico para realizar la búsqueda de las coordenadas de los puntos del área donde no se cumplen las restricciones. Luego, tomando esos puntos como iniciales se aplica una búsqueda de máximos locales (método de Nelder-Mead). Estos últimos pueden ser los puntos críticos. Cada nuevo punto (restricción) representa una línea adicional en la matriz de programación no lineal. Utilizando los datos de puntos críticos se calculan los coeficientes de la matriz A y la parte derecha de las restricciones (b). En el problema considerado de optimización semi-infinita, las fuentes se reemplazan por el número finito de fuentes virtuales. Así el vector de soluciones aumenta su tamaño $U$ veces. Esta complejidad permite hallar las emisiones aumentadas no solo para cada fuente sino, también, para su contenido disperso. Esto economiza los gastos de disminución del conflicto definido en las normas. En seguida se propone la formulación de la función objetivo (9) del problema SIP con sus restricciones (10) para las fuentes virtuales:

$$
C_{x} \rightarrow \min \left(\pi \sum_{j=1}^{U N} m_{j} x_{j} \frac{H 2_{j}^{2}}{2 A_{j}}\right)
$$

Donde: $x_{j}$ es la parte sobrante de la potencia de fuente virtual $\mathrm{j} ; m$ es el costo de filtración por unidad de volumen de emisiones. La función objetivo (9) minimiza los costos de purificación de las emisiones totales. El conjunto infinito de restricciones (10) compara las emisiones de todas las fuentes virtuales con la norma de contaminación en cada punto de área:

$g_{s}=\left(\sum_{j=1}^{U N}\left(-A_{j} R_{s, j}^{2}+\left(1-x_{j}\right) H 2_{j}\right)\right)-\operatorname{Norm}\left(\omega_{s}\right) \leq 0$

\section{EXPERIMENTOS NUMERICOS}

La composición de las partículas de emisiones industriales corresponde básicamente al nano rango (0.01 $40 \mu \mathrm{m}$ ), aunque no se excluye la presencia de partículas más grandes del rango tecnológico (hasta $80-100$ $\mu \mathrm{m})$. Las partículas de tamaño PM10 y PM2.5 son particularmente dañinas y difíciles de eliminar del cuerpo y, además, su propagación afecta grandes áreas. Para el caso P10, Vázquez, Beristain y Audelo (2012) estudiaron los registros de la contaminación también haciendo énfasis en las partículas en suspensión.

El área considerada está definida sobre la malla $d[800,400]$, donde se encuentran $N=17$ las fuentes puntuales de emisiones. El coeficiente único de la difusión de la contaminación es $a=0.044$. El área está dividida en $\Omega=12$ zonas, donde operan las normas de seguridad Norm (figura 2). 
Los experimentos numéricos y los gráficos fueron realizados en MATLAB. Este software es apropiado para problemas de optimización semi-infinita ya que, permite trabajar con grandes cantidades de datos a velocidades de cálculo adecuadas. Adicionalmente, la estructura matricial para hacer operaciones conlleva a una reducción significativa en el código del programa. MATLAB también contiene una variedad de los procedimientos de optimización, como por ejemplo, el algoritmo de Nelder-Mead (Zahara y Kao, 2009), útil para la solución de problemas SIP y tiene unas posibilidades de proyectar y generar las funciones neuronales. La potente interfaz gráfica 2D-3D permite visualizar rápidamente no solo los resultados finales sino las iteraciones intermedias. El uso de las funciones neuronales en problemas de ecología industrial proporcionan representaciones de calidad de datos de monitoreo o pronósticos que se usan bastante en la práctica (Henríquez y Palma, 2011).

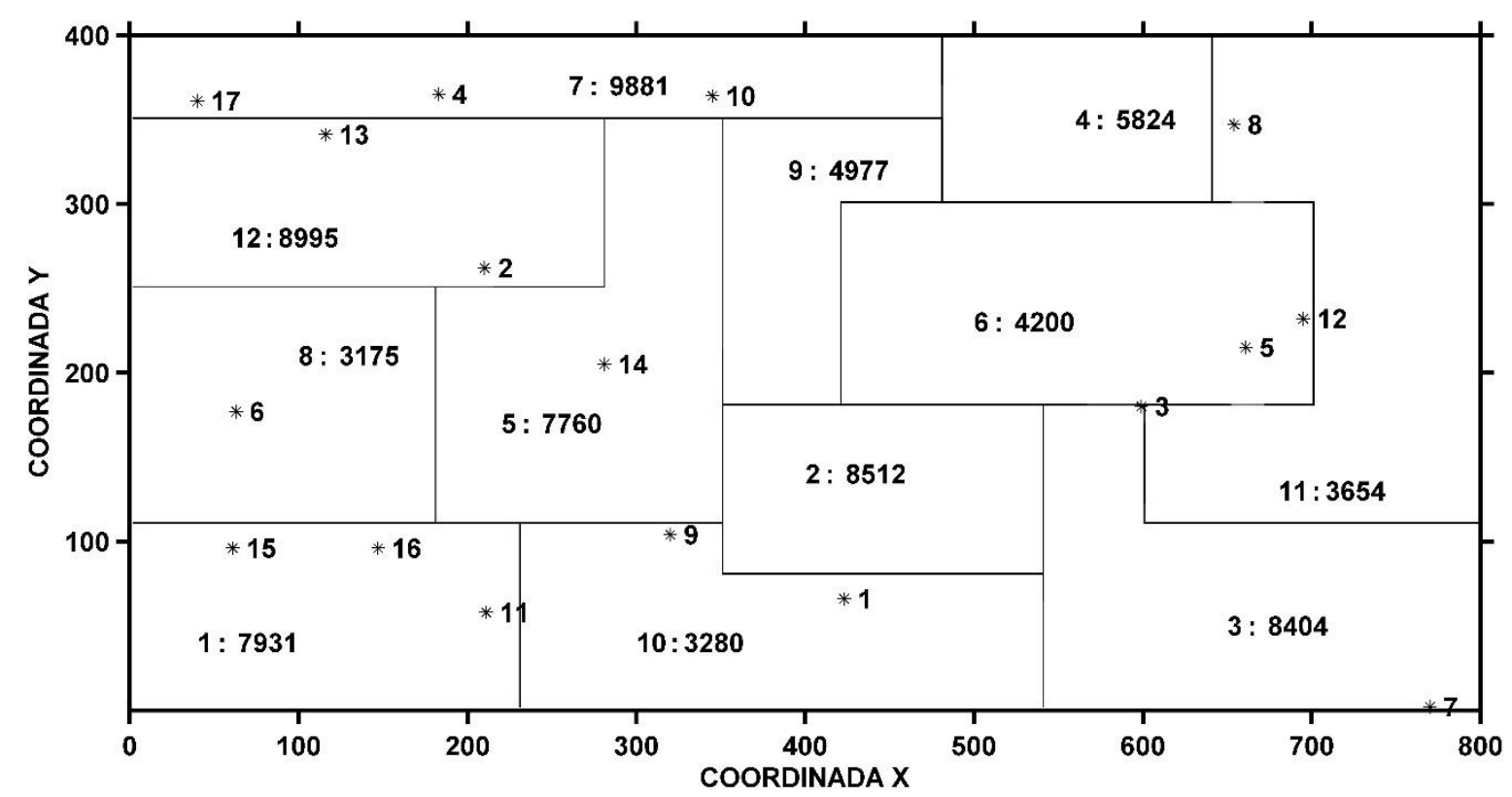

Fig. 2: Mapa de ubicación de las fuentes de contaminación en zonas del área.

Las emisiones de $N$ fuentes conducen a la formación de un entorno de contaminación por partículas en el rango condicional de tamaños 4-67 $\mu \mathrm{m}\left(10^{-6} \mathrm{~m}\right)$.

Se compararon tres opciones de la dispersión de las partículas con los máximos desplazados según la figura 1. El número de los intervalos de dispersión fue $U=3$, por eso cada intervalo contado unió los tres originales. En la tabla 2 están dados los vectores de partículas en los rangos. Los rangos considerados corresponden a las partículas pequeñas (S), medianas (M) y grandes (L). Por ejemplo, en la opción 1, el intervalo 3 es igual a $0.16+0.2+0.25=0.61$ (ver Figura 1). La relación de tamaños de partículas con los rangos contables es uniforme, aunque esto no es necesario. Luego se determinaron los parámetros $N U$ de fuentes virtuales para cada una de las opciones de $U$ (Tabla 3).

Tabla 1: Masas de partículas en los rangos de dispersión de emisiones.

\begin{tabular}{|c|c|c|c|}
\hline \multirow{2}{*}{$\begin{array}{c}\text { Parámetros de } \\
\text { composición dispersa }\end{array}$} & \multicolumn{3}{|c|}{ Los rangos de dimensiones de partículas, $u$} \\
\cline { 2 - 4 } & $1(S)$ & $2(M)$ & $3(L)$ \\
\hline$\mu_{1}$ (opcion 1) & 0.13 & 0.26 & 0.61 \\
\hline$\mu_{2}$ (opcion 2) & 0.17 & 0.55 & 0.28 \\
\hline$\mu_{3}$ (opcion 3) & 0.47 & 0.32 & 0.21 \\
\hline $\begin{array}{c}\text { Dimensión de partículas, } \\
\mu \mathrm{m}\end{array}$ & $4-25$ & $25-46$ & $46-67$ \\
\hline $\mathrm{A}$ & 0.015 & 0.041 & 0.073 \\
\hline $\mathrm{m}$ & 0.0036 & 0.0023 & 0.0013 \\
\hline
\end{tabular}

La estructura de las fuentes virtuales incluye $U=3$ grupos de tamaños de partículas: pequeño (Small), mediano 18-34 (Medium), y grandes 35-51 (Large). Teniendo en cuenta el coeficiente A la zona de dispersión de partículas determina $R_{\max }(S)>R_{\max }(M)>R_{\max }(L)$. Esto conduce a la formación de recubrimientos con un número diferente de capas: a) dentro de radio $R_{\max }(L)$ - capas de tres niveles $(S+M+L)$; b) dentro de radio $R_{\max }(M)-R_{\max }(L)$ - capas de dos niveles $(S+M) ; \mathrm{y}$ c) dentro de radio $R_{\max }(S)-R_{\max }(M)$ - capas de un nivel $(S)$. 
En la figura 3 se encuentra la función de la contaminación inicial para la opción 1. Comparando la figura 3 con las normas definidas en la figura 2 se observa un conflicto moderado. Visualmente es posible ver el no cumplimiento de las normas en las zonas $5,6,8,9,10,11$. En la tabla 3 se encuentran las soluciones encontradas por al algoritmo en proporciones de 0 a 1 . Son los vectores de las potencias excesivas $\mathrm{x}$ para las tres opciones de dispersión (tabla 2).

Tabla 2: Potencias de las fuentes virtuales para las tres opciones.

\begin{tabular}{|c|c|c|c|c|c|c|c|c|}
\hline \multicolumn{9}{|c|}{ Potencias H1 } \\
\hline \multicolumn{3}{|c|}{ Opcion 1} & \multicolumn{3}{|c|}{ Opción 2} & \multicolumn{3}{|c|}{ Opción 3} \\
\hline$S(1-17)$ & $M(18-34)$ & $L(35-51)$ & $S(1-17)$ & $M(18-34)$ & $L(35-51)$ & $S(1-17)$ & $M(18-34)$ & $L(35-51)$ \\
\hline 197 & 460 & 940 & 225 & 669 & 637 & 374 & 510 & 551 \\
\hline 334 & 781 & 1596 & 382 & 1135 & 1081 & 635 & 866 & 936 \\
\hline 105 & 245 & 500 & 120 & 356 & 339 & 199 & 271 & 293 \\
\hline 380 & 889 & 1817 & 435 & 1293 & 1231 & 723 & 986 & 1066 \\
\hline 166 & 388 & 794 & 190 & 565 & 538 & 316 & 431 & 466 \\
\hline 413 & 966 & 1975 & 473 & 1405 & 1338 & 786 & 1072 & 1159 \\
\hline 435 & 1018 & 2080 & 498 & 1480 & 1409 & 828 & 1129 & 1221 \\
\hline 473 & 1105 & 2258 & 540 & 1607 & 1530 & 899 & 1226 & 1325 \\
\hline 285 & 665 & 1360 & 325 & 968 & 921 & 541 & 738 & 798 \\
\hline 53 & 124 & 254 & 61 & 180 & 172 & 101 & 138 & 149 \\
\hline 145 & 338 & 691 & 165 & 492 & 468 & 275 & 375 & 406 \\
\hline 577 & 1349 & 2757 & 660 & 1962 & 1868 & 1097 & 1497 & 1618 \\
\hline 96 & 225 & 461 & 110 & 328 & 312 & 183 & 250 & 270 \\
\hline 522 & 1220 & 2493 & 597 & 1774 & 1689 & 992 & 1353 & 1463 \\
\hline 340 & 795 & 1626 & 389 & 1157 & 1101 & 647 & 882 & 954 \\
\hline 629 & 1471 & 3007 & 720 & 2140 & 2037 & 1196 & 1632 & 1764 \\
\hline 49 & 116 & 236 & 57 & 168 & 160 & 94 & 128 & 139 \\
\hline
\end{tabular}

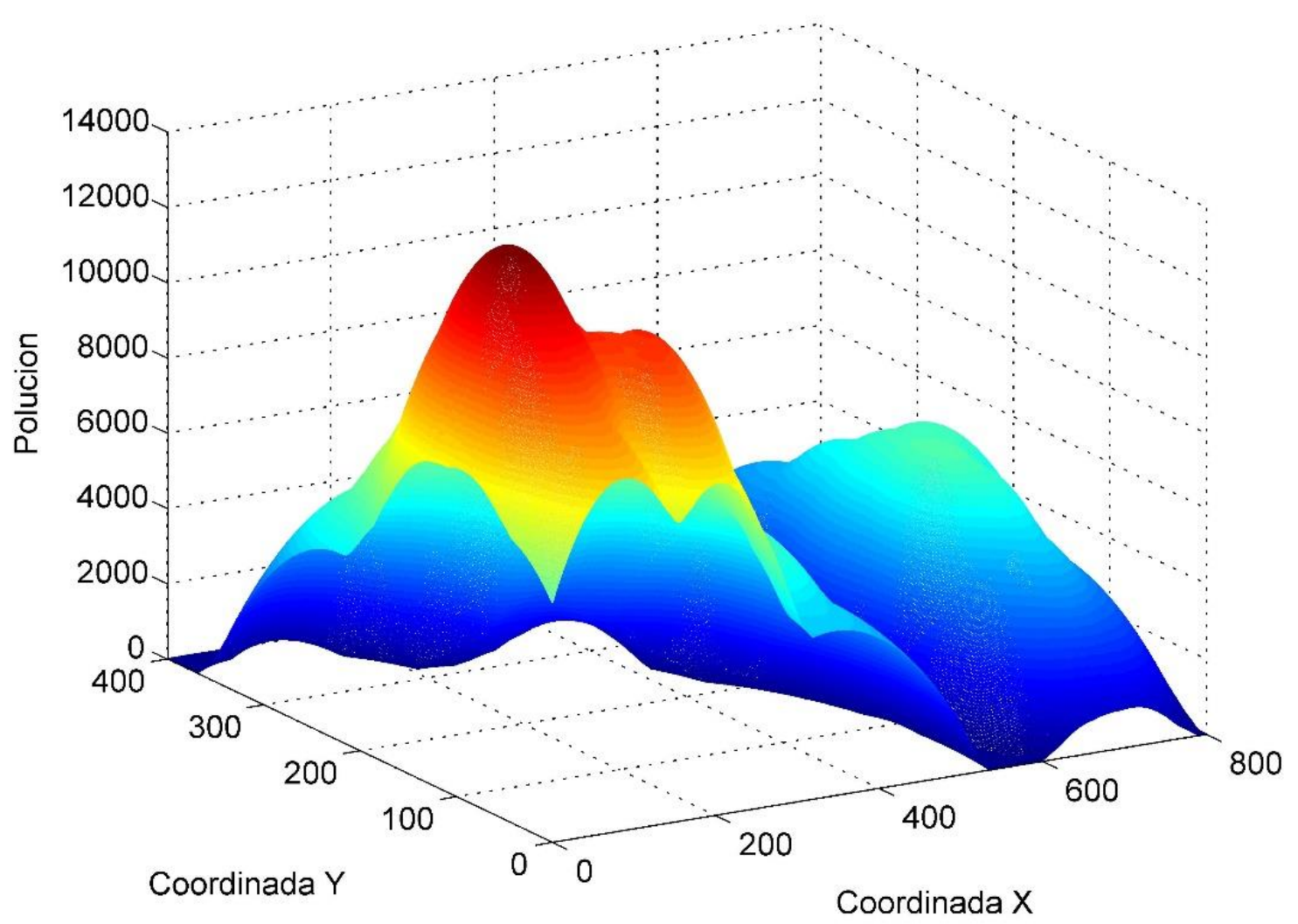

Fig. 3: Función de contaminación inicial por fuentes virtuales (opción 1). 
Cada elemento de vector de soluciones se encuentra en el intervalo $0 \leq x \leq 1$. El '0' significa que la situación está cumpliendo por completo las normas existentes de las zonas y no es necesario revisar (disminuir) las potencias de las fuentes. Al contrario, ' 1 ' significa que las potencias de esas fuentes están totalmente por fuera de las normas y tienen que ser eliminadas del mapa. Los demás valores intermedios entre 0 y 1 muestran el porcentaje de disminución necesaria de contaminación de algunas fuentes. En la práctica, la disminución de la contaminación puede ser aplicada utilizando filtración de las poluciones entre otras tecnologías correspondientes a cada caso. En las figuras 4-6 están las áreas de observación luego de optimización para las opciones 1-3 de dispersión. Las opciones 2 y 3 se parecen entre sí, al contrario, la primera opción tiene diferencias significativas con ellas.

Tabla 3: Las soluciones óptimas para fuentes virtuales para las tres opciones.

\begin{tabular}{|c|c|c|c|c|c|c|c|c|}
\hline \multicolumn{9}{|c|}{ Vector $x$} \\
\hline \multicolumn{3}{|c|}{ Opcion 1} & \multicolumn{3}{|c|}{ Opción 2} & \multicolumn{3}{|c|}{ Opción 3} \\
\hline$S(1-17)$ & $M(18-34)$ & $L(35-51)$ & $S(1-17)$ & $M(18-34)$ & $L(35-51)$ & $S(1-17)$ & $M(18-34)$ & $L(35-51)$ \\
\hline 0 & 0 & 0 & 0 & 0 & 0 & 0 & 0 & 0 \\
\hline 0 & 1 & 1 & 0 & 1 & 1 & 0 & 1 & 0.457 \\
\hline 0 & 0 & 0 & 0 & 0 & 0.315 & 0 & 0 & 0 \\
\hline 0 & 0 & 1 & 0 & 0 & 0 & 0 & 1 & 0.452 \\
\hline 0 & 0 & 0 & 0 & 0 & 0 & 0 & 0 & 0 \\
\hline 0 & 0 & 0.366 & 0 & 0 & 1 & 0 & 0 & 1 \\
\hline 0 & 0 & 1 & 0 & 0 & 1 & 0 & 0 & 1 \\
\hline 0 & 1 & 1 & 0 & 1 & 1 & 0 & 1 & 1 \\
\hline 0 & 0 & 0 & 0 & 0.575 & 1 & 0 & 0.977 & 1 \\
\hline 0 & 0 & 0 & 0 & 0 & 0 & 0 & 0 & 0.964 \\
\hline 0 & 0 & 0.343 & 0 & 0.264 & 1 & 0 & 0.225 & 1 \\
\hline 0 & 0 & 0 & 0 & 0 & 1 & 0 & 0.176 & 1 \\
\hline 0 & 0 & 0 & 0 & 0 & 0 & 0 & 0 & 0 \\
\hline 0 & 1 & 1 & 0 & 1 & 1 & 0.817 & 1 & 1 \\
\hline 0 & 0 & 1 & 1 & 1 & 1 & 1 & 1 & 1 \\
\hline 0 & 0.086 & 1 & 0 & 0.722 & 1 & 0 & 1 & 1 \\
\hline 0 & 0 & 0 & 0 & 0 & 0 & 0 & 0 & 0 \\
\hline
\end{tabular}

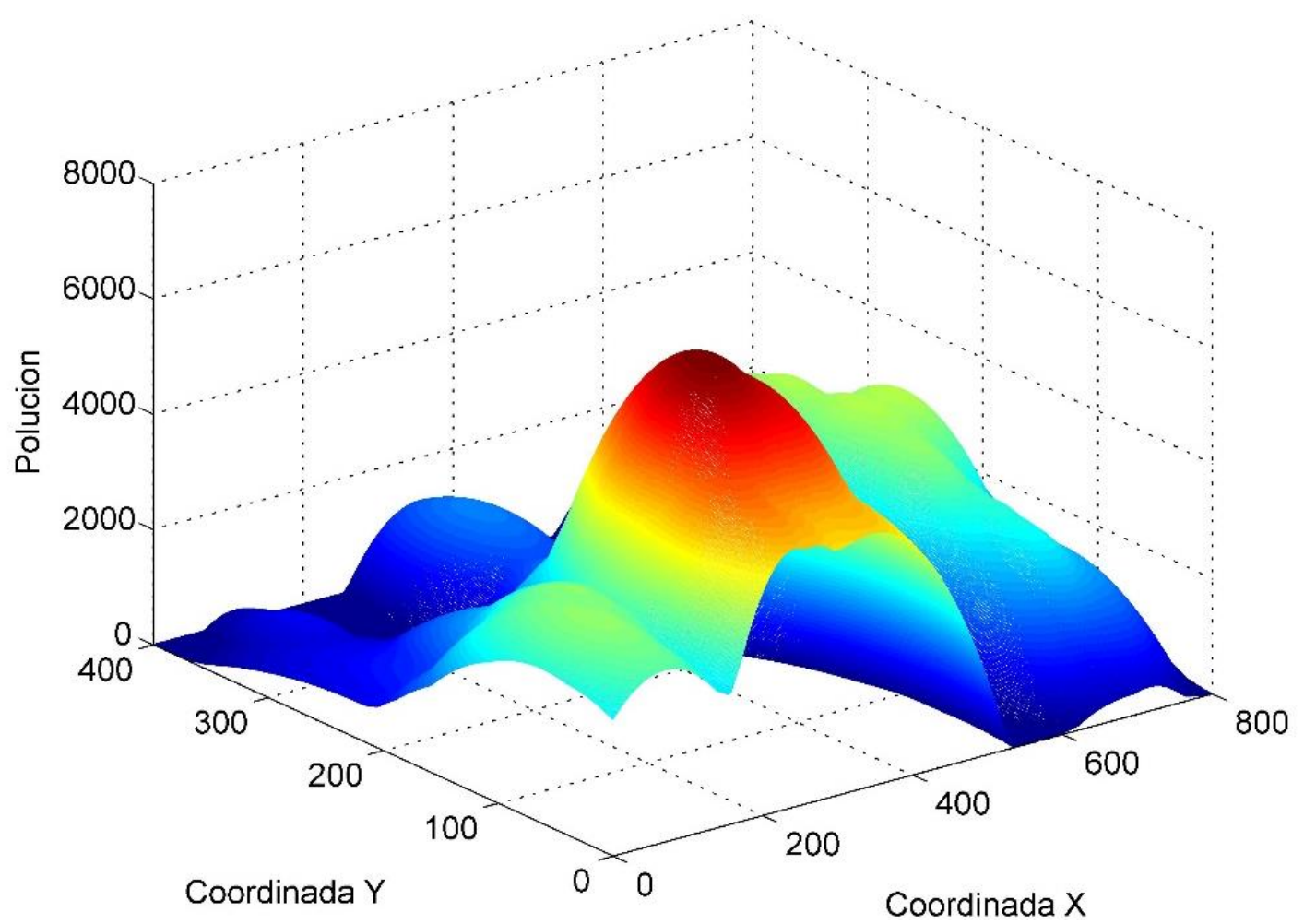

Fig. 4: Contaminación óptima del área por las fuentes virtuales en la opción 1. 


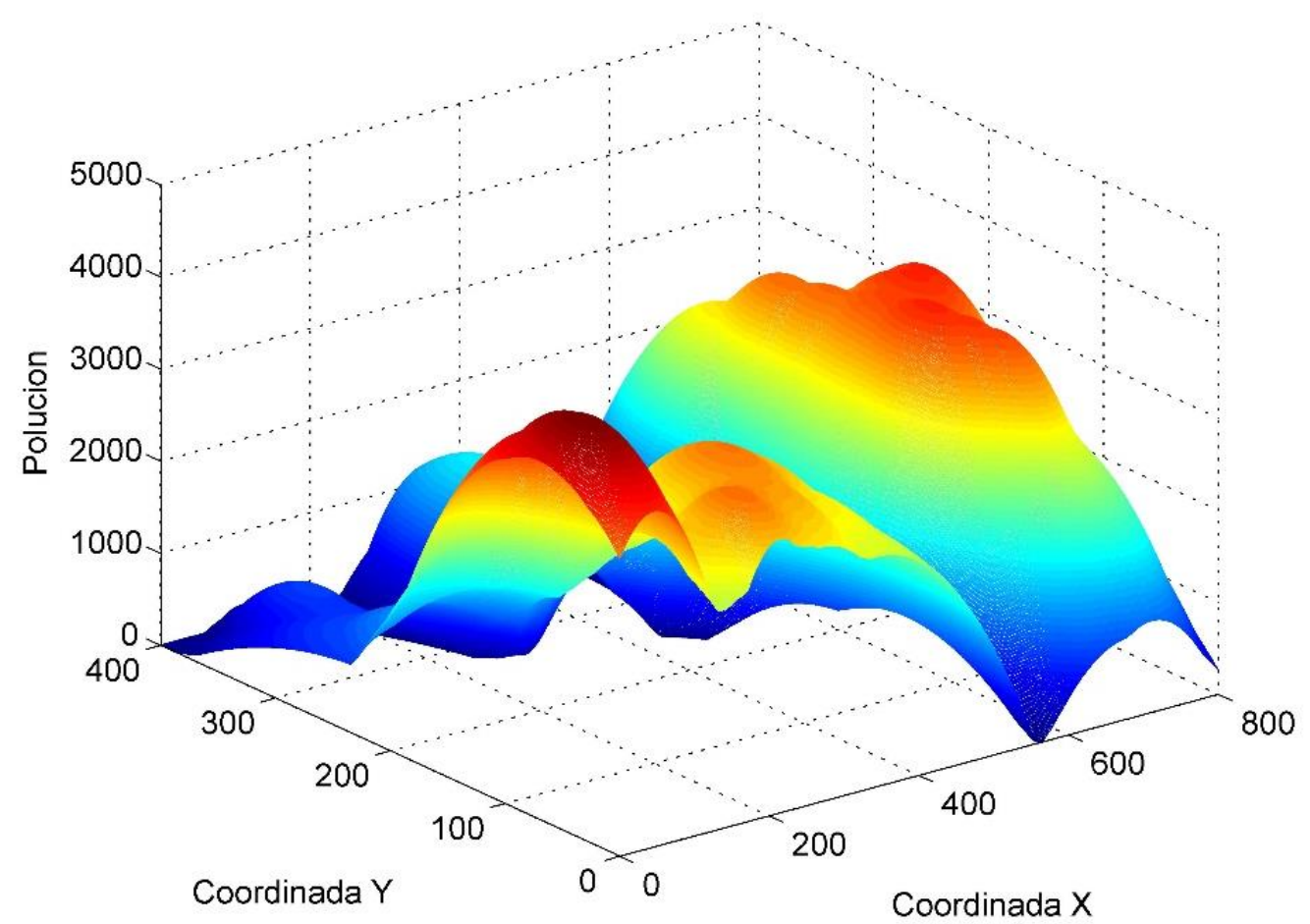

Fig. 5: Contaminación óptima del área por las fuentes virtuales en la opción 2.

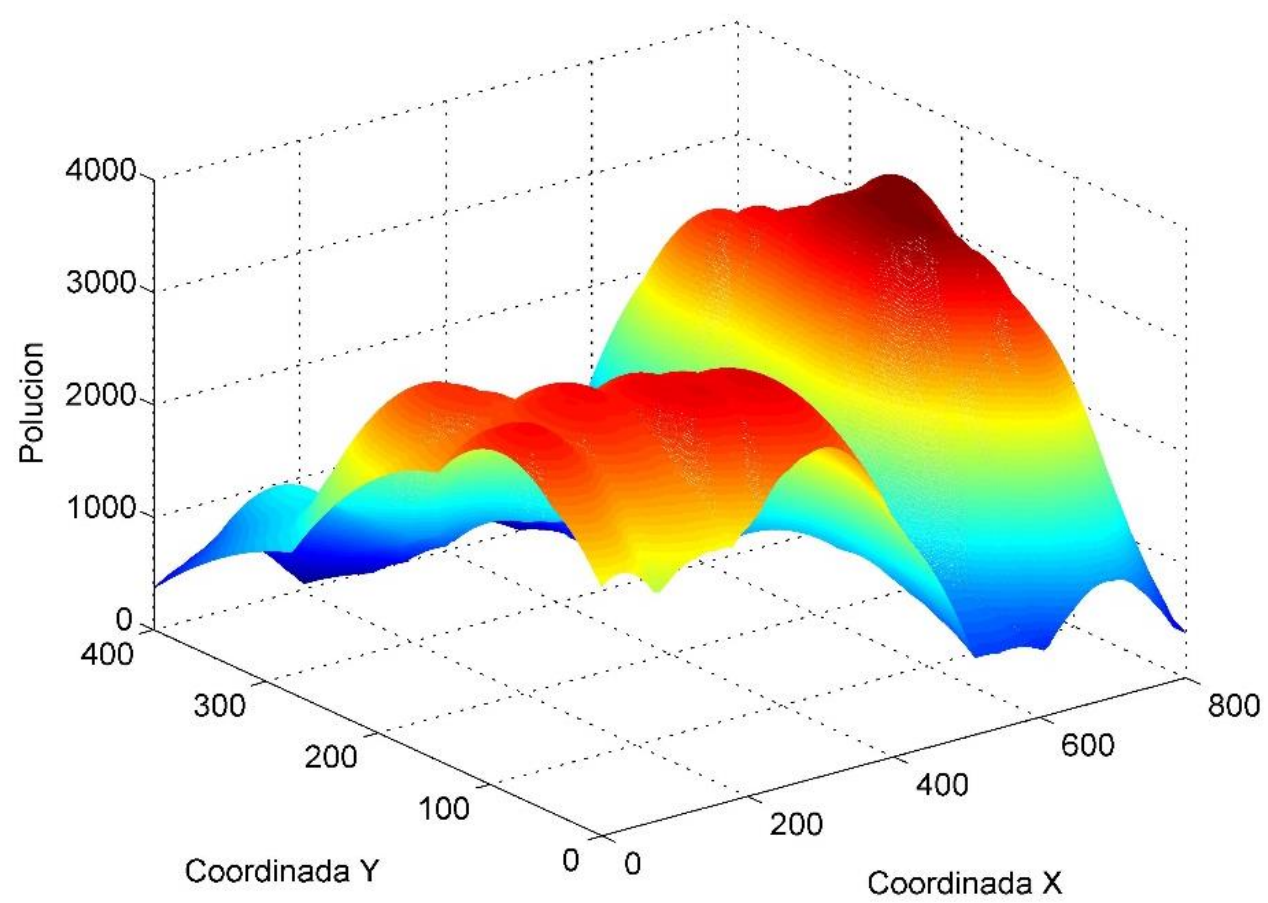

Fig. 6: Contaminación óptima del área por las fuentes virtuales en la opción 3.

Los valores óptimos de la función objetivo según el contenido disperso de cada opción se calcularon como los siguientes C (x) corresponde a: 1) Para la Opción 1 1261654, 2) para la Opción 2 a 1695655, y 3) para la Opción 3 a 1710356.La función objetivo que corresponde a la solución óptima x no solo expresa los costos de purificación, sino que también contiene información adicional. Se puede separar su valor y tener la información para cada fuente y por tamaños de partículas, lo que es interesante en práctica. En la figura 7 se muestran los costos de reducción de producción (y dejar producir) según los resultados de optimización para todas las opciones de contenido de dispersión. También se exhibe por separado los valores de la función objetivo para cada una de los 17 fuentes. 


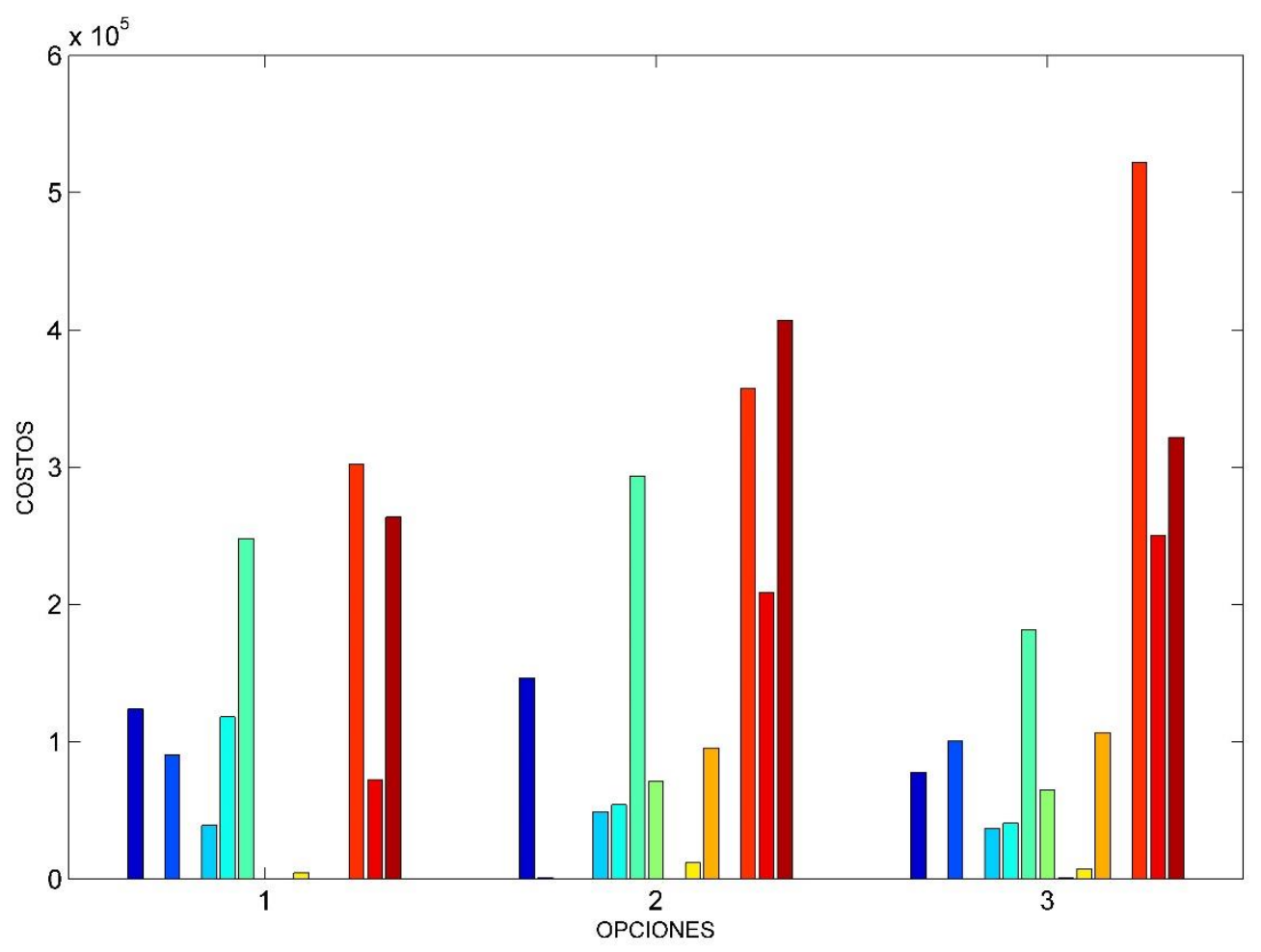

Fig. 7: Costos (perdidas) por fuentes de contaminación.

Los resultados obtenidos muestran que es importante no solo tener en cuenta el rango inicial general de los tamaños de las partículas, sino que también se deben considerar los diferentes tipos de distribución de partículas de contaminación. Es importante resalta que al aumentar la discretización del área controlada, el crecimiento del número de intervalos contables o la cantidad de fuentes virtuales, la velocidad del algoritmo disminuye notablemente, lo que requiere medios informáticos más potentes.

La función objetivo permite incluir formulaciones económicas más complejas. En este trabajo se consideraron las emisiones de un grupo de fuentes que tienen la composición de dispersión similar. Es posible trabajar el esquema donde el grupo se parte según las dispersiones diferentes. En total, cada fuente podría tener su propio tipo de dispersión.

\section{CONCLUSIONES}

En este estudio, se introduce por primera vez el parámetro de dispersión de partículas de contaminantes en la optimización de las emisiones (simultáneas) de una serie de fuentes industriales. Se obtuvieron soluciones que logran cumplir la variedad de los estándares de contaminación existentes en cada punto del área controlada. Esto distingue el problema y su optimización de los comunes, donde solo se mejoran los criterios integrales para una región.

Los resultados del trabajo permiten concluir que es importante considerar el parámetro de dispersión de partículas de emisiones en problemas de contaminación del aire ya que aumenta la exactitud de los mapas de contaminación de varias fuentes. En el artículo se estudiaron tres tamaños diferentes de partículas contaminantes en donde la función objetivo de las partículas más grandes (46-67 $\mu \mathrm{m})$ obtuvo el valor más alto de 1710356, evidenciando la mayor capacidad de reducción de costos de purificación y menores costos de optimización para las partículas pequeñas y de tamaño medio. Dado que el parámetro de dispersión se introduce por primera vez en esta investigación, no es posible comparar resultados con los publicados por otros investigadores. La optimización semi-infinita de aproximaciones externas que se utiliza permite encontrar una solución óptima que cumple las normas de todas las zonas para cada punto del área, detallando los resultados según cada fuente, su aspecto y composición de partículas de polución.

\section{REFERENCIAS}

Duque, J.F., Sobre los Efectos de la Contaminación Atmosférica en la Salud, Basado en el texto Clean the air for children, UNICEF (2016) 
Fedosov, V., A. Fedossova y O. Buitrago, Outer Approximation Algorithms to Solve Semi-infinite Environmental Pollution Problems, INGE-CUC, 14(1), 19-29 (2018)

Fedosov, V. y A. Fedossova, Numerical Optimization of Pollution in 3D Area by Groups of Sources, Nanoengineering, 7, 43-48 (2014)

Fedosov V. y A. Fedossova, Multiparameter Optimization Problems in Conflict Industrial Ecolog,; Information Technologies, 23(10), 721 - 727 (2018)

Gomez, A., A. Fedossova y R. Britto, A Numerical Solution for the Air Pollution Control Problem in 3D, Dyna, 84(200), 342350 (2017)

Gouveia, N., L.J. Washington y otros doce autores, Effects of Air Pollution on Infant and Children Respiratory Mortality in Four Large Latin-American Cities, doi: org/10.1016/j.envpol.2017.08.125, Environmental Pollution, 232, 385-391 (2018)

Graff Zivin, J. y M. Neidell, The Impact of Pollution on Worker Productivity, American Economic Review, 102(7), $3652-73$ (2012)

Henríquez, M. y P. Palma, Control Automático de Condiciones Ambientales en Domótica usando Redes Neuronales Artificiales, doi: 10.4067/S0718-07642011000300014, Información Tecnológica, 22(3), 125-139 (2011)

Kohn, R.E., A Cost-effectiveness Model for Air Pollution Control with a Single Stochastic Variable, Journal of the American Statistical Association, 67(337), 19-22 (1972)

Künzli, N., R. Kaiser y otros doce autores, Public-health Impact of Outdoor and Traffic-related Air Pollution: a European Assessment, The Lancet, 356(9232), 795 - 801 (2000)

Normas GOST (Rusia) 17.2.3.02-2014 Normas de Emisiones de Contaminación Permitidas para las Empresas Industriales. OAO "NII Atmosfera", Aprobado 2015-07-01, Rusia (2014)

Rojano, R., H. Arregoces y G. Restrepo, Composición Elemental y Fuentes de Origen de Partículas Respirables (PM10) y Partículas Suspendidas Totales (PST) en el Área Urbana de la Ciudad de Riohacha, Colombia, doi: /10.4067/S071807642014000600002, Información Tecnológica, 25(6), 3-12 (2014)

Vázquez C., F. Beristain y E. Audelo, Caracterización del Patrón de Comportamiento de las Partículas en Suspensión en el Valle de México, doi: 10.4067/S0718-07642012000300002, Información Tecnológica, 23(3), 3-10 (2012)

Vaz, A.I.F. y E.C. Ferreira, Air Pollution Control with Semi-infinite Programming, Applied Mathematical Modelling, 33(4), 1957-1969 (2009)

Volkov, Y.V. y S.K. Zavriev, A General Stochastic Outer Approximations Method, SIAM Journal on Control and Optimization, 35(4), 1387-1421 (1997)

Zahara, E. e Y.T. Kao, Hybrid Nelder-Mead Simplex Search and Particle Swarm Optimization for Constrained Engineering Design Problems, Expert Systems with Applications, 36 (2), 3880-3886 (2009)

Zaitseva N.V., I.V. May, A.A. Maks y S.Y. Zagorodnov, Análisis de la Composición Dispersa y Componente del Polvo para Evaluación de la Exposición de la Población en las Zonas de Influencia de Fuentes Estacionarias Industriales, Higiene y Saneamiento, 5, 19-23 (2013)

Zavriev S.K., N.M. Novikova y A. Fedossova, Algoritmo Estocástico para Solucionar Problemas Convexos de Optimización Semi-infinita con Restricciones Igualdades y Desigualdades, Vestnik MSU, 15(4), 30-35 (2000) 
